\title{
CONSTRUÇÃO DE UM MICROGERADOR FOTOVOLTAICO APLICADO AO PENEIRAMENTO DE RESÍDUOS DA CONSTRUÇÃO CIVIL
}

\author{
Paulo Alex Nacif Lube \\ Mestre pelo Programa de Pós-Graduação em Desenvolvimento Local do \\ Centro Universitário Augusto Motta, UNISUAM, Rio de Janeiro, RJ. \\ paulolube@gmail.com \\ Kátia Eliane Santos Avelar \\ Doutora em Ciências pela Universidade Federal do Rio de Janeiro, UFRJ. \\ Docente e Pesquisadora do Programa de Pós-Graduação em Desenvolvimento Local no \\ Centro Universitário Augusto Motta, UNISUAM \\ katia.avelar@gmail.com \\ Patricia Maria Dusek \\ Doutora em Ciências pela Universidade Federal do Rio de Janeiro, UFRJ. \\ Docente e Pesquisadora do Programa de Pós-Graduação em Desenvolvimento Local no \\ Centro Universitário Augusto Motta, UNISUAM \\ patricia.dusek@unisuam.edu.br
}

\section{RESUMO}

A demanda por geração de energia com a utilização de fontes renováveis está crescendo rapidamente. A energia solar fotovoltaica se caracteriza como uma alternativa aos sistemas energéticos convencionais e poluentes, por ser uma energia limpa, renovável e com a vantagem de possibilitar a produção no próprio local de consumo. Este estudo teve por objetivo apresentar a construção de um microgerador fotovoltaico destinado ao uso em peneira separadora de resíduos da construção civil (RCC). O projeto elétrico para a construção do microgerador levou em consideração informações de consumo, o potencial solar do local de instalação e a autonomia de funcionamento de uma peneira elétrica. $\mathrm{O}$ microgerador foi construído em uma plataforma móvel, com potência suficiente para atender à demanda energética de uma peneira elétrica, que é um equipamento utilizado nos aterros de triagem e transbordo para a separação de grãos finos e aproveitamento de RCC. A construção do microgerador fotovoltaico propiciou a infraestrutura necessária para o fornecimento de energia para uma peneira elétrica em um aterro de transbordo e triagem. Tal tecnologia tornou o processo de aproveitamento de RCC mais sustentável, com ganhos ambientais, econômicos, sociais e, consequentemente, contribuiu para o desenvolvimento local.

Palavras-chave: Energia Fotovoltaica. Resíduo sólido. Desenvolvimento local. 


\title{
CONSTRUCTION OF A PHOTOVOLTAIC MICROGENERATOR APPLIED TO THE SCREENING OF CIVIL CONSTRUCTION WASTE
}

\begin{abstract}
The demand for power generation using renewable sources is growing rapidly. Photovoltaic solar energy is characterized as an alternative to conventional and polluting energy systems, as it is clean, renewable and with the advantage of enabling production at the place of consumption. This study aimed to present the construction of a photovoltaic microgenerator intended for use in a separator sieve for construction waste. The electrical project for the construction of the microgenerator took into account consumption information, the solar potential of the installation site and the autonomy of operation of an electric sieve. The microgenerator was built on a mobile platform, with enough power to meet the energy demand of an electric sieve, which is used in sorting and transshipment landfills for the separation of fine grains and the use of construction waste. The construction of the photovoltaic microgenerator provided the necessary infrastructure for the supply of energy for an electric sieve in an overflow and sorting landfill. Such technology made the process of using construction waste more sustainable, with environmental, economic, social gains and, consequently, contributed to local development.
\end{abstract}

Keywords: Photovoltaic energy. Solid waste. Local development. 


\section{INTRODUÇÃO}

Os impactos ambientais relativos à geração de energia elétrica têm adquirido notoriedade na mídia e na vida da população em geral. Atentos à essa questão, estudos buscam desenvolver maneiras para minimizar esses impactos e tornar os sistemas geradores de energia mais competentes e sustentáveis.

Desta forma, para Cunha (2019), dentre as fontes energéticas alternativas, tem-se em destaque a energia solar, da qual sua viabilidade se caracteriza no vasto crescimento da capacidade instalada nos últimos anos, tanto no Brasil como no mundo. Assim, essa fonte energética já desempenha um papel valoroso no processo de transição da matriz energética do planeta, que vai na direção de uma produção compatível com as questões ambientais, atuais e futuras.

Diante disso, compreende-se que a energia solar é a energia adquirida por meio do calor e da luz do sol, a qual é capturada e utilizada por diversas tecnologias, como a energia fotovoltaica, energia hélio térmica e arquitetura solar. A energia solar é caracterizada como energia de fonte renovável e sustentável (PEREIRA; MENDES, 2018). De acordo com esse autor, a energia fotovoltaica é a energia elétrica gerada por meio da conversão direta da luz, através da radiação solar. Assim, quanto maior a incidência de luz, maior a geração de eletricidade, podendo também ser adquirida em dias nublados e/ou chuvosos.

A obtenção da energia elétrica por meio da conversão da energia solar é realizada por meio do efeito fotovoltaico. Portanto, Pereira e Mendes (2018) ressaltam que o componente principal de um sistema fotovoltaico é a célula, e, é onde acontece a principal função do sistema, a conversão da radiação solar em energia elétrica, por meio do efeito fotovoltaico.

A energia fotovoltaica apontou um crescimento substancial nos últimos anos, bem como reduções consideráveis de custos, elevando assim o desafio em se estabelecerem modelos de matrizes energéticas integradas, combinando diferentes fontes energéticas e vislumbrando o fato da energia solar ser um modelo dominante a longo prazo (PIETZCKER et al., 2014).

Segundo Ursaia et al. (2012), países desenvolvidos como Alemanha, Japão e Estados Unidos, que buscam crescer no mercado, apresentam destaque no sistema solar 
fotovoltaico, sendo que em 2004 foram responsáveis por 94\% da capacidade instalada no ano.

De acordo com a Agência Internacional de Energia - IEA (IEA, 2017), as energias renováveis especulam representar dois terços do investimento global em usinas de geração de energia até o ano de 2040, transformando-se na fonte de energia com o menor custo de geração. Assim, o rápido estabelecimento da energia solar fotovoltaica, liderada pela China e Índia, contribuem a energia solar a ser a maior fonte geradora de energia com baixas emissões de carbono até o ano de 2040, quando a participação de todas as energias renováveis chegará a $40 \%$ do total de energia produzida no planeta.

Segundo Novosel et al. (2015), a Jordânia é um país que enfrenta diversos problemas com o meio ambiente relacionados à demanda por energia. Este país apresentase como o quarto país mais privado de água, com um consumo anual de apenas $145 \mathrm{~m}^{3}$ per capita, apesar de ser privilegiada em recursos de energia eólica e solar, $99 \%$ da eletricidade produzida é proveniente de combustíveis fósseis importados, resultando em altas emissões de carbono e inúmeros problemas de abastecimento (NOVOSEL et al., 2015).

Dessa maneira, considerando-se a boa representatividade do Brasil no quesito da grande radiação solar em todas as regiões, este apresenta-se como um país privilegiado, tendo em vista que é do sol que se obtém a energia solar fotovoltaica (VILLALVA; GAZOLI, 2015).

Portanto, na mesma linha, Lima Júnior (2018) aponta que a utilização da energia luminosa do sol é uma das opções mais promissoras para integrar a matriz energética brasileira, que apesar do seu potencial, fornece atualmente menos de $1 \%$ da energia elétrica gerada anualmente no Brasil. A alta incidência de radiação solar ao longo do ano no país, em especial na região nordeste, se deve à sua localização na região intertropical do planeta onde esse recurso energético é abundante.

Contudo, os estudos do uso de energia solar costumam se restringir a grandes empreendimentos de geração conectada à rede elétrica, ou casos de sistemas isolados em locais remotos. Estudos relacionados ao uso desta tecnologia em equipamentos utilizados em Aterros de Transbordo e Triagem (ATT) de resíduos da construção civil (RCC) são raros, principalmente, em países em desenvolvimento como o Brasil.

No ATT, uma das etapas do processo de separação dos resíduos sólidos é realizada em uma peneira elétrica. A peneira é alimentada com energia de baterias automotivas. Essas 
são recarregadas com a utilização de motor a Diesel com elevado custo, ineficiência energética e emissão de $\mathrm{CO}_{2}$. $\mathrm{O}$ interesse pela pesquisa partiu da premissa em atender demanda econômica, social, política e ambiental.

O estudo está alinhado com a ODS 7, Energia Acessível e Limpa, que trata do acesso às fontes de energia, principalmente as renováveis (ONU, 2015). Pelo exposto, o presente trabalho teve como objetivo desenvolver um microgerador individual de energia elétrica, através de placas fotovoltaicas, aplicado em uma peneira elétrica, separadora de resíduos sólidos oriundos da construção civil, possibilitando assim a autonomia do equipamento, a geração de energia, através de fonte renovável e, consequente, redução de custos e aumento de renda dos trabalhadores que operam no ATT contribuindo, assim, para o desenvolvimento socioambiental dos trabalhadores.

\section{MATERIAIS E MÉTODOS}

Os materiais utilizados na construção do protótipo foram os seguintes: 2 barras cantoneiras de $5 / 8 \times 1 / 8 ; 2$ barras chatas de $5 / 8 \times 1 / 8 ; 1$ chapa galvanizada $262 \times 1 ; 4$ Gonzos de ferro 3/8; 2 barras quadradas 3/8; 2 rodas de borracha 10; 1 roda de borracha direcional; 2 Hastes reguláveis de antena parabólica; $1 \mathrm{~m}^{2}$ de adesivos; 1 Bateria estacionária 12v 450ah; 2 placas fotovoltaicas 150WP - células de policristalino; 1 controlador de carga 30A 12/24v PWM; 20 m de cabo flexível solar 6mm; 2 Conectores MC4; 2 Conectores MC4 Multibranch; 2 conectores de polo de bateria; Haste de aterramento; 2 disjuntores Bipolar Painel 440v 32a CC; DPS CC 600v Dispositivo Proteção Surtos 20ka 40ka Bipolar; Caixa de disjuntores. A execução do projeto seguiu o princípio de equilíbrio entre viabilidade técnica versus viabilidade econômica.

\subsection{Desenvolvimento do Projeto Elétrico}

O projeto elétrico do microgerador foi desenvolvido a partir das informações de consumo e autonomia de funcionamento da peneira elétrica da marca NORTEC. A peneira possui dois motores vibratórios do fabricante OLI - Modelo MVE 200 com potência de 160Watts (W), com 12Volts (V) de tensão CC e corrente de 13,3 Ampères (A) cada um deles, tendo em média 2,5 horas de funcionamento por dia. 


\subsection{Recurso Solar}

Foram utilizados os dados da irradiação diária, disponibilizada pelo Laboratório de Modelagem e Estudos de Recursos Renováveis de Energia (LABREN). Irradiação Global Horizontal - Sistema LABREN - INPE, Duque de Caxias, RJ de 4706 Watts hora por metro quadrado $\left(\mathrm{Wh} / \mathrm{m}^{2} \mathrm{dia}\right)$.

\subsection{Dimensionamento do Painel Fotovoltaico}

O dimensionamento do painel fotovoltaico foi idealizado de acordo com o consumo e horas de funcionamento da peneira. A potência foi definida pela razão do consumo diário, pelo produto das horas de sol pico e fator de captação. A quantidade de painéis foi determinada pela razão da potência do arranjo fotovoltaico, pela potência do painel a ser utilizado (SOLARIZE SERVIÇOS EM TECNOLOGIA AMBIENTAL LTDA, 2017).

Para esse microgerador, foi determinado um arranjo com dois painéis ligados em paralelo. No arranjo fotovoltaico foram utilizados Módulos Policristalinos de Alta eficiência do modelo RSM36-6-150P da marca Risen. Esses módulos são produzidos com uma tecnologia avançada de processamento de células, gerando uma eficiência de mais de $15,5 \%$.

Para o cálculo do controlador de carga, levou-se em consideração a potência total do arranjo fotovoltaico e a tensão do sistema. Foi utilizado um Controlador de Carga programável, da marca Epever, com tensão de operação de 12/24V, Tecnologia PWM, corrente de carga e descarga da bateria de 30A e tensão máxima de 50Vcc. Todas as medições de tensão e corrente realizadas nos testes foram obtidas por esse medidor digital. O controlador de carga desconecta a carga após sua tensão atingir o valor máximo de descarga e com isso preserva a vida útil da bateria.

\subsection{A escolha da Bateria}

A bateria utilizada para servir como acumulador de energia do sistema fotovoltaico é da Marca MAX POWER, Modelo MP 3000 - 400Ah estacionária. O banco de bateria(s) deve 
ser de no mínimo 297Ah, proporcionando autonomia diária de 2,5 horas de funcionamento da peneira, para três dias sem recarga.

\subsection{Desenvolvimento do Protótipo}

\subsubsection{Modelo Computacional}

Para o desenvolvimento do modelo computacional foi utilizado o programa Autodesk AutoCAD ${ }^{\circledR} 2020$, conforme figura 1, abaixo.

Figura 1 - Modelo Computacional do microgerador

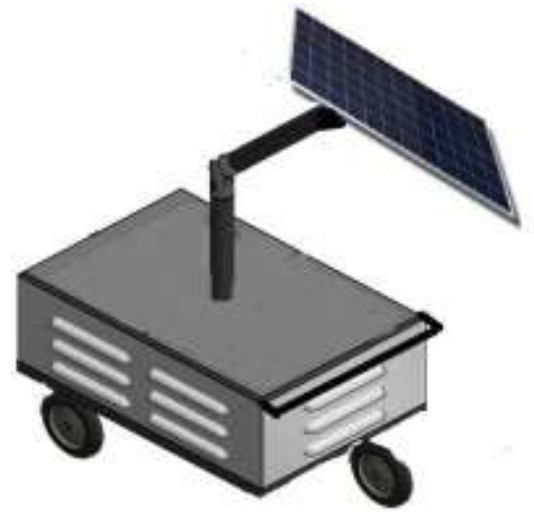

Fonte: Arquivo dos autores, 2020.

2.5.2 Instalação do microgerador

O microgerador foi conectado aos motores da Peneira, utilizando cabo Bipolar com seção de $6 \mathrm{~mm}^{2}$.

\section{RESULTADOS E DISCUSSÕES}

\subsection{Teste de Eficiência da Bateria}

O armazenamento de energia, geralmente composto por bateria(s), é fundamental para fornecer energia à carga nos períodos sem incidência solar. Para garantir um maior 
rendimento no armazenamento da energia gerada pelos painéis, foram realizados alguns testes na tentativa de encontrar a eficiência da bateria quando submetida à situação de carga e descarga.

Para a garantia da confiabilidade do teste, a bateria foi totalmente carregada. A tensão inicial da bateria $\mathrm{Vm}=12,3(\mathrm{~V})$ com aproximadamente $70 \%$ da carga. A recarga da bateria do microgerador foi iniciada no dia 29 de agosto de 2019, às $10 \mathrm{~h}$, pausada às $15 \mathrm{~h}$ do mesmo dia. Reiniciada às $10 \mathrm{~h}$ do dia 30 de agosto e finalizada às $13 \mathrm{~h}$, foram anotados os valores da corrente elétrica fornecida pelas placas fotovoltaicas ligadas em paralelo em intervalos de 1 hora. Tempo total de recarga de 9 horas, conforme tabela 1.

Tabela 1: Levantamento da carga da bateria em diferentes intervalos de tempo

\begin{tabular}{lc}
\hline HORÁRIO & A (AMPERES) \\
\hline $29 / 08 / 2019$ 10:00 & 14,0 \\
\hline $29 / 08 / 2019$ 11:00 & 15,1 \\
\hline $29 / 08 / 201912: 00$ & 16,0 \\
\hline $29 / 08 / 2019$ 13:00 & 15,9 \\
\hline $29 / 08 / 2019$ 14:00 & 15,3 \\
\hline $29 / 08 / 2019$ 15:00 & 14,3 \\
\hline $30 / 08 / 201910: 00$ & 14,1 \\
\hline $30 / 08 / 2019$ 11:00 & 15,4 \\
\hline $30 / 08 / 2019$ 12:00 & 15,9 \\
\hline $30 / 08 / 2019$ 13:00 & 16,0 \\
\hline MÉDIA & 15,2 \\
\hline
\end{tabular}

Fonte: Elaborada pelos autores, 2020.

O teste de eficiência das baterias durante um ciclo profundo e lento de descarga se deu a partir das informações contidas no manual técnico da bateria (tabela 2). 
Tabela 2: Tensões de configuração para bateria 12V Max Power

\begin{tabular}{|c|c|}
\hline CARACTERÍSTICAS & PARÂMETRO DE CONFIGURAÇÃo \\
\hline Tensão de Equalização & 14,20 a $14,40 \mathrm{~V}$ \\
\hline Tensão de Flutuação & 13,20 a $13,38 \mathrm{~V}$ \\
\hline
\end{tabular}

Fonte: Elaborada pelos autores, 2020.

Com a bateria completamente carregada, realizou-se sua descarga de maneira que a carga foi drenada com uma corrente de 27,6 A. Conforme catálogo do fabricante, a corrente deve ser de 33A para ciclos de 10 horas. A Bateria em C (10) = regime de descarga em 10 horas com Ibat $=33$ A. Utilizaram-se os Motores da peneira no mesmo regime de carga C (10). De acordo com as informações técnicas da bateria, essa descarga se daria em aproximadamente 12 horas e 23 minutos. O processo foi iniciado às 7 h02 e a bateria atingiu seu valor mínimo às $18 \mathrm{~h} 09$ do mesmo dia, totalizando $11 \mathrm{~h} 07$.

\subsection{Testes de Funcionamento - Geração e Consumo}

Após o teste de recarga, foi adotado um regime diário de levantamento de dados sobre o clima, a utilização da peneira e o microgerador. Os resultados obtidos estão descritos no gráfico 1 . Os dados apresentados mostram que a disponibilidade de energia pelo microgerador foi suficiente para a utilização na peneira durante o seu funcionamento diário. 
Gráfico 1: Peneiramento, consumo e geração de energia com o Microgerador fotovoltaico

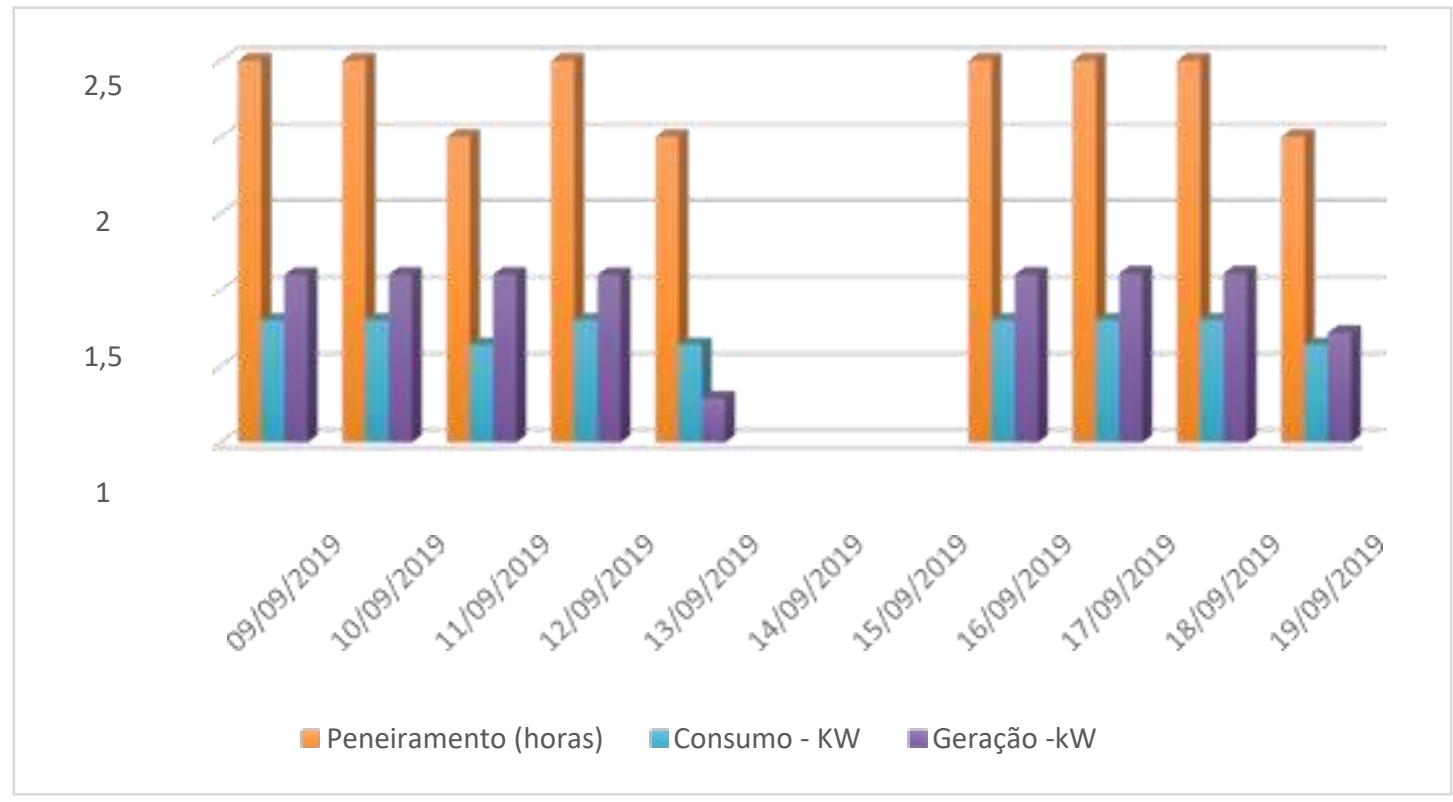

Fonte: Elaborado pelos autores, 2020.

A Área de Transbordo e Triagem (ATT) consiste em uma estação intermediária entre o gerador do resíduo e a destinação final. Os resíduos são recebidos, passam por um processo de triagem, são acondicionados por seu tipo e classe e, posteriormente, são enviados para um local adequado, observando normas operacionais especificadas, de modo a evitar danos ou riscos à saúde pública e à segurança e a minimizar os riscos ambientais (CETESB, 2019).

O material recolhido é separado. O resíduo de origem mineral (concreto, argamassa, alvenaria, dentre outros) é encaminhado para aterros de inertes, o rejeito é levado para aterros sanitários e o resíduo reaproveitável é comercializado. No caso das novas ATTs, os resíduos são levados para aterros particulares. A separação dos resíduos de origem mineral é realizada através da granulometria, que é a distribuição em porcentagem, dos diversos tamanhos de grãos. Em uma das etapas, utiliza-se uma peneira elétrica para separação dos agregados finos (menores que 6,0 mm de diâmetro).

Segundo a NBR6502/95 os pedregulhos grossos têm grãos comprometidos entre 20,0 e 60,0 mm, os médios têm grãos comprometidos entre 0,6 e $20,0 \mathrm{~mm}$ e os finos têm grãos comprometidos entre 2,0 e 6,0 mm. 


\subsection{Desempenho do microgerador}

\subsubsection{Disponibilidade de produção de energia}

De acordo com os dados de irradiação do sistema LABREN, o local onde o microgerador está em funcionamento têm em média $4.706 \mathrm{Wh} / \mathrm{m}^{2}$ dia de disponibilidade de produção de energia.

\subsubsection{Entrega de energia}

Verificou-se a energia que foi realmente produzida pelo sistema para consumo. Pode ser mostrada anual, mensal ou diária, por hora. Com base nos dados dos testes de funcionamento realizados, chegou-se à média de produção de 0,983KWh/dia.

\subsubsection{Desempenho de Energia}

Representa a porcentagem entre a energia produzida da energia esperada. Assim, conseguiu-se ter uma razão da produção de energia gerada pelo microgerador versus a energia estimada que a instalação tem capacidade de gerar.

\subsection{Análise da viabilidade econômica}

Foi realizada uma análise das opções disponíveis no mercado, a tecnologia, a viabilidade e o valor dos equipamentos necessários para execução do projeto. O valor investido foi de $R \$ 3.561,74$.

De acordo com os fabricantes e especificações de uso, os equipamentos têm individualmente seu tempo previsto de uso. Se estabelecermos como parâmetro a vida útil de 4 anos da bateria, como referência para a primeira substituição, a geração de energia média diária dos testes e o valor da tarifa da concessionária de energia, pode-se concluir que o microgerador tem resultado negativo, pois o valor do investimento inicial não retornará com a produção de energia ao longo do tempo de vida útil do microgerador. 
Levando-se em consideração que para a utilização da energia da concessionária seria necessário um investimento de aproximadamente 12 mil reais em infraestrutura (conversor $\mathrm{CA} / \mathrm{CC}$, cabos, postes ou eletrodutos, itens de conexão e distribuição), somados à tarifa de consumo, o investimento no microgerador se torna viável e se tem um resultado positivo, com uma economia inicial de $70 \%$.

\section{CONSIDERAÇÕES FINAIS}

$\mathrm{Na}$ avaliação final, este projeto conseguiu sucesso ao atingir seus objetivos. Procurou-se projetar um sistema que atendesse seguramente aos parâmetros necessários, para a operação da peneira é possível concluir que o microgerador projetado atende às especificações técnicas e de funcionamento.

A mobilidade do protótipo possibilita adaptá-lo às características do terreno e, também, à captação da radiação solar, com possibilidade de ajuste para a direção do sol ao longo do dia.

O emprego energético da conversão fotovoltaica causa diferentes impactos econômicos, dependendo do sistema em que ele é gerado. Em ATT's, esse modelo permite a autossuficiência energética em locais com grandes áreas e pouca infraestrutura.

O presente trabalho serve como incentivo para a ampliação dessa solução energética no Brasil, integrando o uso sustentável dos recursos naturais renováveis, com o uso racional e eficiente de energia. No entanto, para que isso ocorra, é necessário incentivo.

Outro aspecto importante é a grande vantagem da utilização desse tipo de tecnologia, que está diretamente vinculada ao ganho ambiental, quando comparada com a tecnologia de grupos geradores de combustão.

$\mathrm{Na}$ análise financeira da tecnologia, o resultado é extremamente favorável ao uso da energia fotovoltaica offgrid. De acordo com as características dos motores da peneira e a infraestrutura encontrada no ATT, os resultados indicam que incialmente se tem uma economia de $70 \%$ do investimento necessário, se fosse utilizada a energia da concessionária.

Os ganhos ambiental e social são intangíveis quando se agrega tecnologia e conhecimento científico, somados aos ganhos produtivo e de eficiência nas atividades do ATT.

Os aspectos sociais, econômicos e ambientais atendidos nesse projeto indicam que 
é possível e viável unir o desenvolvimento local e a sustentabilidade.

\section{REFERÊNCIAS}

CETESB. Companhia Ambiental do Estado de São Paulo. Sistema Estadual de Gerenciamento de Resíduos Sólidos. Informações sobre as áreas de destinação. São Paulo, SP, 2019. Disponível em: https://cetesb.sp.gov.br/sigor/informacoes-sobre-as-areas-dedestinacao/\#1507859735781-d52477de-3433. Acesso em: 13 jul. 2019.

CUNHA, Eduardo Argou Aires. Aspectos históricos da energia eólica no Brasil e no mundo. Revista Brasileira de Energias Renováveis, v. 8, n. 4, p. 689- 697, 2019.

IEA. Agência Internacional de Energia. Energy Technology Perspectives. 2017. ISBN: 978-9264-27597-3.

LIMA JÚNIOR, Claudemiro et al. Energia solar: metodologia para avaliação do local de instalação de sistema fotovoltaico fomentando a educação ambiental. Revbea, São Paulo, v. 13, n. 3, p. 233-244, 2018.

NOVOSEL, T. et al. Integration of renewables and reverse osmosis desalination e Case study for the Jordanian energy system with a high share of wind and photovoltaic, Energy, v. 92, p. 270-278, 2015. Http://dx.doi.org/10.1016/j.energy.2015.06. 057.

ONU. Transformando Nosso Mundo: a Agenda 2030 para o Desenvolvimento Sustentável. 2015. Disponível em: https://nacoesunidas.org/wp-content/uploads/2015/10/agenda2030pt-br.pdf. Acesso em: 01 ago. 2020.

PEREIRA, Fabiana Luzia; MENDES, Marina Alves. 0 uso de energia solar fotovoltaica como alternativa à redução da fatura de energia elétrica em blocos universitários. 2018. Trabalho de Conclusão de Curso (Bacharelado em Engenharia Civil) - Universidade do Sul de Santa Catarina, Tubarão, 2018.

PIETZCKER, R.; STETTER, D.; MANGER, S.; LUDERER, G. Using the sun to decarbonize the power sector: The economic potential of photovoltaic and concentrating solar power. Applied Energy, v. 135, n. 15, p. 704-720, 2014.

SOLARIZE SERVIÇOS EM TECNOLOGIA AMBIENTAL LTDA. Curso de energia solar, projetista de sistema fotovoltaico conectado à rede. Ministrado por: Hans Rauschmayer e Ronaldo Rocha. Rio de Janeiro, 2017.

URSAIA, Guilherme Crippa et al. O Quadro Político e Institucional do Setor Energético Brasileiro. UNISUL de Fato e de Direito: Revista Jurídica da Universidade do Sul de Santa Catariana, v. 2, n. 4, 2012. Disponível em: http://www.portaldeperiodicos.unisul.br/index.php/U_Fato_Direito/article/view/1072/8 93. Acesso em: 12 jul. 2019. 
VILLALVA, Marcelo Gradella; GAZOLI, Jonas Rafael. Energia Solar Fotovoltaica: conceitos e aplicações. 2. ed. São Paulo: Erica, 2015. 